University of Wollongong

Research Online

Faculty of Engineering and Information

Faculty of Engineering and Information

Sciences - Papers: Part A

Sciences

$1-1-2012$

On the dosimetric effect and reduction of inverse consistency and transitivity errors in deformable image registration for dose accumulation

\author{
Edward T. Bender \\ University Of Wisconsin-Madison \\ Nicholas Hardcastle \\ University of Wollongong, nhardc@uow.edu.au \\ Wolfgang A. Tome \\ University of Wollongong, wtome@uow.edu.au
}

Follow this and additional works at: https://ro.uow.edu.au/eispapers

Part of the Engineering Commons, and the Science and Technology Studies Commons

Research Online is the open access institutional repository for the University of Wollongong. For further information contact the UOW Library: research-pubs@uow.edu.au 


\title{
On the dosimetric effect and reduction of inverse consistency and transitivity errors in deformable image registration for dose accumulation
}

\begin{abstract}
Purpose: Deformable image registration (DIR) is necessary for accurate dose accumulation between multiple radiotherapy image sets. DIR algorithms can suffer from inverse and transitivity inconsistencies. When using deformation vector fields (DVFs) that exhibit inverse-inconsistency and are nontransitive, dose accumulation on a given image set via different image pathways will lead to different accumulated doses. The purpose of this study was to investigate the dosimetric effect of and propose a postprocessing solution to reduce inverse consistency and transitivity errors. Methods: Four MVCT images and four phases of a lung 4DCT, each with an associated calculated dose, were selected for analysis. DVFs between all four images in each data set were created using the Fast Symmetric Demons algorithm. Dose was accumulated on the fourth image in each set using DIR via two different image pathways. The two accumulated doses on the fourth image were compared. The inverse consistency and transitivity errors in the DVFs were then reduced. The dose accumulation was repeated using the processed DVFs, the results of which were compared with the accumulated dose from the original DVFs. To evaluate the influence of the postprocessing technique on DVF accuracy, the original and processed DVF accuracy was evaluated on the lung 4DCT data on which anatomical landmarks had been identified by an expert. Results: Dose accumulation to the same image via different image pathways resulted in two different accumulated dose results. After the inverse consistency errors were reduced, the difference between the accumulated doses diminished. The difference was further reduced after reducing the transitivity errors. The postprocessing technique had minimal effect on the accuracy of the DVF for the lung 4DCT images. Conclusions: This study shows that inverse consistency and transitivity errors in DIR have a significant dosimetric effect in dose accumulation; Depending on the image pathway taken to accumulate the dose, different results may be obtained. A postprocessing technique that reduces inverse consistency and transitivity error is presented, which allows for consistent dose accumulation regardless of the image pathway followed.
\end{abstract}

\section{Keywords}

reduction, effect, dosimetric, transitivity, consistency, errors, inverse, deformable, image, registration, dose, accumulation

\section{Disciplines}

Engineering | Science and Technology Studies

\section{Publication Details}

Bender, E. T., Hardcastle, N. \& Tome, W. A. (2012). On the dosimetric effect and reduction of inverse consistency and transitivity errors in deformable image registration for dose accumulation. Medical Physics, 39 (1), 272-280. 


\title{
On the dosimetric effect and reduction of inverse consistency and transitivity errors in deformable image registration for dose accumulation
}

\author{
Edward T. Bender \\ Department of Human Oncology, University of Wisconsin-Madison, Madison, Wisconsin 53792 \\ Nicholas Hardcastle \\ Department of Human Oncology, University of Wisconsin-Madison, Madison, Wisconsin 53792 and Centre for \\ Medical Radiation Physics, University of Wollongong, Wollongong, New South Wales 2522, Australia \\ Wolfgang A. Toméa) \\ Department of Human Oncology, University of Wisconsin-Madison, Madison, Wisconsin 53792; Department \\ of Medical Physics, University of Wisconsin-Madison, Madison, Wisconsin 53792; Department of Biomedical \\ Engineering, University of Wisconsin-Madison, Madison, Wisconsin 53792 and Centre for Medical Radiation \\ Physics, University of Wollongong, Wollongong, New South Wales 2522, Australia
}

(Received 18 July 2011; revised 7 November 2011; accepted for publication 17 November 2011; published 21 December 2011)

Purpose: Deformable image registration (DIR) is necessary for accurate dose accumulation between multiple radiotherapy image sets. DIR algorithms can suffer from inverse and transitivity inconsistencies. When using deformation vector fields (DVFs) that exhibit inverse-inconsistency and are nontransitive, dose accumulation on a given image set via different image pathways will lead to different accumulated doses. The purpose of this study was to investigate the dosimetric effect of and propose a postprocessing solution to reduce inverse consistency and transitivity errors. Methods: Four MVCT images and four phases of a lung 4DCT, each with an associated calculated dose, were selected for analysis. DVFs between all four images in each data set were created using the Fast Symmetric Demons algorithm. Dose was accumulated on the fourth image in each set using DIR via two different image pathways. The two accumulated doses on the fourth image were compared. The inverse consistency and transitivity errors in the DVFs were then reduced. The dose accumulation was repeated using the processed DVFs, the results of which were compared with the accumulated dose from the original DVFs. To evaluate the influence of the postprocessing technique on DVF accuracy, the original and processed DVF accuracy was evaluated on the lung 4DCT data on which anatomical landmarks had been identified by an expert.

Results: Dose accumulation to the same image via different image pathways resulted in two different accumulated dose results. After the inverse consistency errors were reduced, the difference between the accumulated doses diminished. The difference was further reduced after reducing the transitivity errors. The postprocessing technique had minimal effect on the accuracy of the DVF for the lung 4DCT images.

Conclusions: This study shows that inverse consistency and transitivity errors in DIR have a significant dosimetric effect in dose accumulation; Depending on the image pathway taken to accumulate the dose, different results may be obtained. A postprocessing technique that reduces inverse consistency and transitivity error is presented, which allows for consistent dose accumulation regardless of the image pathway followed. (C) 2012 American Association of Physicists in Medicine.

[DOI: $10.1118 / 1.3666948]$

Key words: deformable image registration, inverse consistent, transitive, dose accumulation

\section{INTRODUCTION}

Adaptive radiotherapy (ART) aims to use anatomical and functional imaging during the course of radiotherapy treatment to monitor the effect of radiotherapy and adjust the treatment plan if necessary to maintain treatment goals. Deformable image registration (DIR) attempts to provide mapping between volume elements in one image to corresponding volume elements in a second image. In the context of ART, DIR is used to map the dose delivered to the anatomy represented in one image to anatomy presented in a second image. Dose accumulation is then the mapping of multiple dose grids from different frames of reference, such as different images, to one common frame of reference.

There are many automated DIR algorithms that can provide a mapping or a deformation vector field (DVF) between two images. These algorithms can suffer from a lack of inverse consistency and transitivity. Given two images, $I_{A}$ and $I_{B}$, inverse inconsistency is when deforming from $I_{A} \rightarrow I_{B} \rightarrow I_{A}$ produces a result different to $I_{A}$; the difference between the result obtained and the actual image $I_{A}$ is termed the inverse consistency error (ICE). ${ }^{1}$ The ICE can be extended to more than two images, which is referred to as the transitivity error (TE), which was referred to as generalized inverse consistency 
error in Ref. 1. Given three images, $I_{A}, I_{B}$, and $I_{C}$, the deformation from $I_{A} \rightarrow I_{B} \rightarrow I_{C} \rightarrow I_{A}$ will produce a result different to the starting image, $I_{A}$. In this scenario (which uses three images), a set of DVFs that produce the same image as that which was started with (that is, $I_{A}$ ) is defined here to be "three-image transitive." It can be shown mathematically that if a set of inverse-consistent DVFs is three-image transitive, it will also be transitive for any higher number of images (see Lemma in the Appendix).

It is desirable for an algorithm to provide both an inverse consistent and transitive result. There are multiple algorithms that provide an inverse consistent transformation, via the inclusion of an extra term in the optimization equation that minimizes the ICE in addition to the minimization of the difference between the two images and the regularization term. ${ }^{2,5,6}$ However, these inverse consistent methods do not necessarily provide a transitive transformation, ${ }^{3,4}$ since they are primarily derived using data from two images only. Geng et $a l .{ }^{4}$ presented a registration method, whereby data from three images were used to derive the transformation based on a function that included terms to minimize the difference between contours or surfaces as well as ICE and TE. A further report by Christensen and Johnson ${ }^{3}$ provided a mathematical framework to analyze nonrigid transformations based on ICE and TE. Skrinjar et al. ${ }^{7}$ also provided a method for transitive registration by performing the DIR between two images in a series via an arbitrary third, "reference" image where the reference image can be another image in the series or one similar to the images in the series.

There are many commercial and research algorithms currently available that are neither inverse consistent nor transitive but are used for dose accumulation. To the best of the authors knowledge, there have been no investigations to date into the effect of inverse inconsistencies and nontransitivity on dose accumulation. The present study shows the dosimetric effect of inverse consistency and transitivity errors in dose accumulation and presents a DVF postprocessing method to reduce inverse inconsistencies and transitivity errors and their effect on dose accumulation. It is currently an open question whether the presented method also improves the accuracy of dose accumulation; however, this is beyond the scope of this work and will be addressed in a future study.

\section{METHODS AND MATERIALS}

\section{II.A. Effect of inverse consistency and transitivity errors on dose accumulation}

To investigate the effect of inverse consistency and transitivity errors on dose accumulation, a dose accumulation process on two data sets was performed. A research version of the Pinnacle RTPS (v9.100, Philips Radiation Oncology Systems, Fitchburg, WI) was used for all DIR and dose accumulation. The DIR algorithm used was the Fast Symmetric Demons image intensity-based algorithm as implemented in the Insight Toolkit. ${ }^{8}$ This variant of the Demons algorithm is neither inverse consistent nor transitive.

The first data set consisted of four tomotherapy MVCT images from a head and neck treatment, representing the anatomical change over the duration of the treatment course (primarily weight loss and shrinkage.) These were merged with the planning kVCT image, such that the MVCT slices replaced all of their corresponding $\mathrm{kVCT}$ slices using the registration parameters used for daily treatment setup in the TomoTherapy PLANNED ADAPTIVE software (v4.0, TomoTherapy, Middleton, WI). Representative axial slices from the four images are shown in Fig. 1. The dose delivered to the merged image in each treatment fraction was calculated in the PLANNED ADAPTIVE software and exported. All four merged images and their dose grids were then imported into Pinnacle.

DIR was performed between all image sets (12 deformations in total). The region selected for DIR only included the MVCT regions of each merged image so as to minimize artifacts in the registration at MVCT-kVCT interfaces. The dose accumulation process is shown in Fig. 2. Each dose grid represented the dose from one fraction of treatment, with the high dose PTV receiving 2.16 Gy per fraction. To simulate a dose accumulation process over a full treatment, the dose on each image was multiplied by 8 to simulate the use of four images during a treatment course. The DVFs are defined in the target image frame of reference. Therefore, dose accumulation is achieved by looking up the voxel of interest in the target image, obtaining the vector pointing back to the source image and then looking up the dose at the location the vector points to in the source image. Since the vectors do not point exactly to the center of dose voxels, trilinear interpolation between the doses in voxels surrounding the vector destination is used to obtain the dose. Dose was accumulated using 17.28 Gy per image for a total dose of 69.12 Gy to the highdose PTV. The four dose grids were accumulated onto the last image in the series, $I_{D}$ to result in Dose $D^{a c}$. The four dose grids were then accumulated onto $I_{C}$ using the same fractionation scheme to result in Dose $C^{a c}$. The accumulated Dose $C^{a c}$ was then mapped to $I_{D}$ to result in Dose $D^{a c}{ }_{2}$. The difference between Dose $D^{a c}{ }_{1}$ and Dose $D^{a c}{ }_{2}$ was measured using dose volume histograms (DVHs) for target and organ at risk (OAR) structures.

The second data set consisted of four phases from a 4DCT of the thorax from the MD Anderson DIR Lab series. ${ }^{9}$ This data set (\#1 in the series) consists of ten images representing each phase of a 4DCT with respiratory motion visible between each phase. On the $0 \%$ and $50 \%$ phase images, the locations of 300 corresponding image features are given.
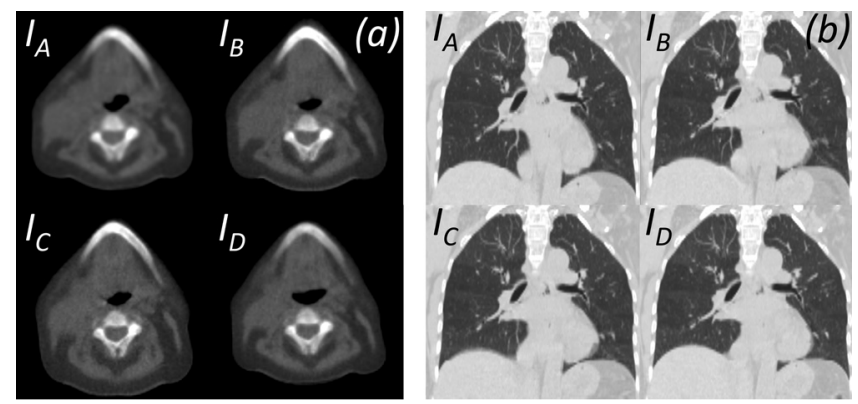

FIG. 1. (a) Axial slices of the head and neck MVCT data and (b) coronal slices of the lung 4DCT data used in the study. 


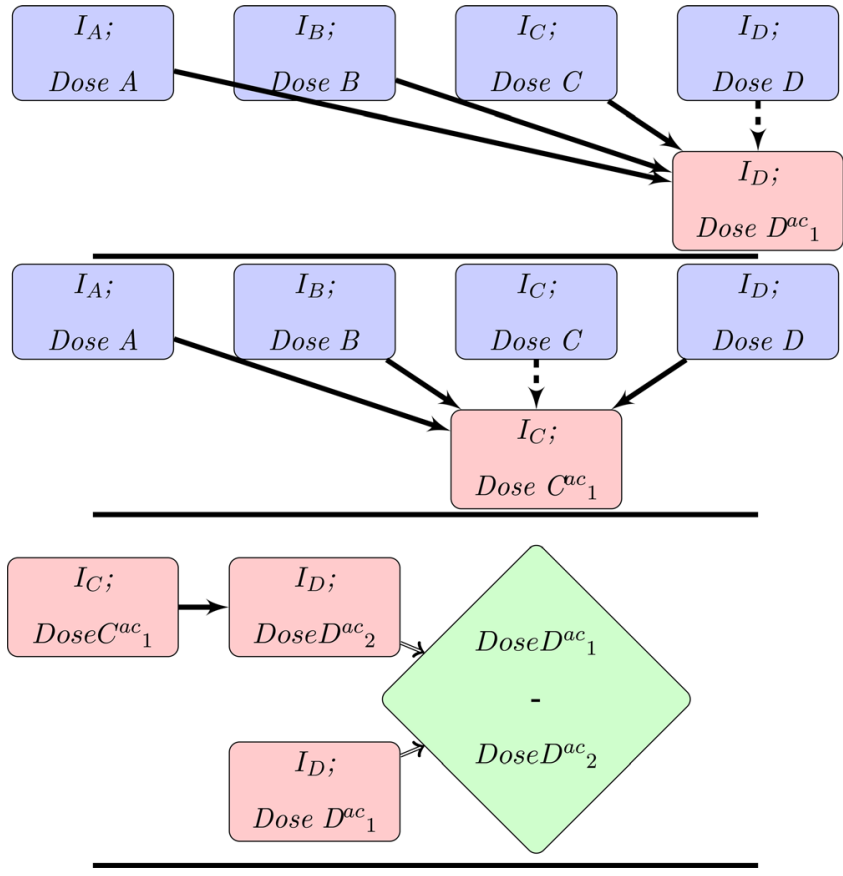

FIG. 2. Flow chart showing the process of dose accumulation to $I_{D}$ via two different image pathways. Solid lines represent accumulation with deformation, dashed lines represent accumulation without deformation.

The $0 \%, 20 \%, 30 \%$, and $50 \%$ phase images were selected for analysis. These images represent the extremes of the deformation and two time points with anatomy approximately evenly distributed between the two extremes. A hypothetical lung tumor residing on the diaphragm of the left lung was outlined and a density of $1 \mathrm{~g} / \mathrm{cm}^{3}$ was assigned to the target volume. A 9 field 3DCRT plan was created to deliver 64 Gy to the target volume in 32 fractions. On each image, the dose from eight fractions of the treatment was calculated. The dose calculated on each image was accumulated and compared using the same process used in Fig. 2 and used for the H\&N MVCT data set.

\section{II.B. Postprocessing technique to reduce inverse consistency and transitivity errors}

Postprocessing was performed with in-house developed applications using MATLAB 2010b (The MathWorks, Natick, MA). All DVFs were processed to reduce ICE using following equation:

$$
\vec{\triangle}_{i j}^{I C}=\frac{1}{2}\left(\vec{\triangle}_{i j}+\vec{\triangle}_{j i}^{-1}\right),
$$

where $\vec{\triangle}_{i j}^{I C}$ is the inverse consistent DVF from $I_{i}$ to $I_{j}, \vec{\triangle}_{i j}$ is the original DVF from $I_{i}$ to $I_{j}$ and $\vec{\triangle}_{j i}^{-1}$ is the inverted, original DVF from $I_{j}$ to $I_{i}$.

DVF inversion for Eq. (1) was achieved using the triScatteredInterp function in MATLAB. The DVFs used in this study are defined in the target image coordinate frame. Therefore, inversion requires obtaining the vectors at each point, which are defined on a regular grid and reversing their direction, which leads to the vectors being defined on an irregular grid. The triScatteredInterp function then aligns these vectors according to a regular grid, in our case that of the source image. This is the same method of inversion utilized by Bender and Tomé. ${ }^{1}$ The Fast Symmetric Demons implementation used in this study is based on diffeomorphic deformations. Diffeomorphic transformations were introduced by Dupuis et al. and are smooth deformations that preserve topology of the image. ${ }^{10}$ The necessary condition for DVF invertibility is that the Jacobian is non-negative. The DVFs obtained using the Fast Symmetric Demons algorithm had very few voxels with a negative Jacobian, thus were effectively invertible. ${ }^{11}$

The result of Eq. (1) may not be exactly inverse consistent due to rounding errors in the inversion process. Therefore, this calculation was performed three times using the previous iteration's result as the input for the current iteration. All DVFs were then further processed to reduce TE using Eq. (2), where $N$ is the number of image sets $(N=4$ in the presented cases).

$$
\begin{aligned}
& \vec{\triangle}_{i k}^{T 3}=\frac{1}{N-1}\left(\vec{\triangle}_{i k}^{I C}+\sum_{j \in S} \vec{\triangle}_{j k}^{I C} \circ \vec{\triangle}_{i j}^{I C}\right) \\
&=\frac{1}{N-1}\left(\vec{\triangle}_{i k}^{I C}+\sum_{j \in S} \vec{\triangle}_{i j k}^{I C}\right), \\
& S=\{s: s \in\{1, \ldots N\} \wedge s \neq i \wedge s \neq k\},
\end{aligned}
$$

where $\vec{\triangle}_{i k}^{T 3}$ is the third-order transitive deformation map from $I_{i}$ to $I_{k}, \vec{\triangle}_{i k}^{I C}$ is the inverse consistent DVF from $I_{i}$ to $I_{k}$ and $\vec{\triangle}_{i j k}^{I C}$ is the inverse consistent DVF from $I_{i}$ to $I_{k}$ via each other $I_{j}$ in the set of images $S . \vec{\triangle}_{i j k}^{I C}$ is defined as $\vec{\triangle}_{i j k}^{I C} \equiv \vec{\triangle}_{j k}^{I C} \circ \vec{\triangle}_{i j}^{I C}$. The composition operator $\circ$ is used which is the composition of two DVFs. That is, $\vec{\triangle}_{A} \circ \vec{\triangle}_{B}$ is the vectors in $\vec{\triangle}_{A}$ applied to the locations of the vectors in $\vec{\triangle}_{B}$. The shift operator as defined in Bender and Tomé ${ }^{1}$ is used to calculate the composition of two DVFs. The shift operator is required because the DVFs are defined at different points in space. If one wants to add two vector fields together they must be operating on the same point in space. Thus, if one wants to perform the operation $\vec{\triangle}_{2} \circ \vec{\triangle}_{1}$ then one must shift $\vec{\triangle}_{1}$ to the same coordinate frame as $\vec{\triangle}_{2}$. A full description of the shift operator is given in the Appendix of Bender and Tomé. ${ }^{1}$

Forming the average given in Eq. (2) has the effect of combining all DVFs available between images $I_{i}$ and $I_{k}$, including "second order" deformations where $I_{i}$ is first deformed to $I_{j}$, before deformation to $I_{k}$. Equation (2) may introduce inverse consistency errors, therefore, the resulting DVFs from Eq. (2) must be processed using one iteration of Eq. (1). Similarly to processing with Eq. (1), the result of Eq. (2) is not exactly transitive after just one iteration, therefore, this process was performed for three iterations, processing with Eq. (1) before performing the next iteration.

Note that Eq. (2) is the combination of the DVF to be processed plus the composition of $I_{i}$ to $I_{k}$ via a third image in the series $I_{j}$, allowing improved consistency when transforming from $I_{i}$ to $I_{k}$ via an intermediary $I_{j}$. This is termed third order transitivity. It is shown in the appendix that if DVFs in 
a series are third order transitive, then they are transitive for all higher orders. This mathematical principle, therefore, limits the number computations required to obtain transitive maps. For the remainder of this report, the terms inverse consistency error reduced (ICER) and transitivity error reduced (TER) will be used to denote DVFs that have been processed with Eq. (1) and iteratively with Eqs. (2) and (1), respectively.

\section{II.C. Effect of the postprocessing technique on the consistency and accuracy of deformation}

The inverse consistency error (ICE) was calculated for the original, ICER and TER DVFs for the image set $\left\{I_{j}\right\}_{j \in\{A, B\}}$ using following equation:

$$
I C E_{i j}: I_{i} \ni \xi \mapsto\left\|\left(\mathbf{1}-\vec{\triangle}_{j i} \circ \vec{\triangle}_{i j}\right) \xi\right\| \in \Re,
$$

where $I C E_{i j}$ is the ICE between $I_{i}$ and $I_{j},\|\bullet\|$ denotes the Euclidian norm when $I_{i}$ is interpreted as a vector space, $\mathbf{1}$ is the identity matrix, $\vec{\triangle}_{i j}$ is the DVF from $I_{i}$ to $I_{j}$, and $\vec{\triangle}_{j i}$ is the DVF from $I_{j}$ to $I_{i} \cdot \vec{\triangle}_{j i} \circ \vec{\triangle}_{i j}$ is $\vec{\triangle}_{i j}$ applied to $\vec{\triangle}_{j i}$. Hence, $I C E_{i j}$ is a map from $I_{i}$ into real numbers $\Re$.

In practice, Eq. (3) is simply the composition of the two DVFs representing the forward and reverse directions. The composition is calculated again using the shift operator as defined in Bender and Tomé. ${ }^{1}$ Similarly, the TE was calculated for the original, ICER and TER DVFs for the image set $\left\{I_{j}\right\}_{j \in\{A, B, C\}}$ using following equation:

$$
T E: I_{i} \ni \xi \mapsto\left\|\left(\mathbf{1}-\vec{\triangle}_{k i} \circ \vec{\triangle}_{j k} \circ \vec{\triangle}_{i j}\right) \xi\right\| \in \Re,
$$

where $\vec{\triangle}_{i j}$ is the DVF from $I_{i}$ to $I_{j}, \vec{\triangle}_{j k}$ is the DVF from $I_{j}$ to $I_{k}$, and $\vec{\triangle}_{k i}$ is the DVF from $I_{k}$ back to $I_{i}$. When computing Eq. (4), the shift operator is again used as defined in Bender and Tomé. ${ }^{1}$

The dose accumulation process presented in Fig. 2 was repeated for both the H\&N MVCT data and the lung 4DCT data using the ICER DVFs and then repeated again using the TER DVFs. The doses Dose $D^{a c}$ and Dose $D^{a c}$ were compared using dose volume histograms.

The lung 4DCT data set contains 300 corresponding anatomical landmarks on the $0 \%$ and $50 \%$ phase images. Using these 300 corresponding landmarks, the accuracy of the original, ICER and TER DVFs were calculated as the magnitude of difference vector between the vector pointing from a given landmark in the $0 \%$ phase image to its corresponding landmark in the $50 \%$ phase image and the vector pointing from the landmark in the $0 \%$ phase image to its DIR calculated position in the $50 \%$ phase image. An analysis of variance (ANOVA) was performed to ascertain influence of the processing technique on the registration error, comparing the results from ICER DVFs and TER DVFs with the original DVFs. The statistics toolbox in MATLAB was used for the ANOVA calculation with a threshold for statistical significance of $p<0.05$.

\section{RESULTS}

The ICE for the DVFs for the H\&N image set $\left\{I_{j}\right\}_{j \in\{A, B\}}$ is shown in Fig. 3. The ICE is effectively removed after three iterations of Eq. (1). The TE is shown in Fig. 4 for the DVFs for the H\&N image set. The TE is reduced with each successive application of Eq. (2) followed by Eq. (1), however, the TE is never completely removed. Figure 5 shows the mean 3D ICE and TE for the H\&N image sets $\left\{I_{j}\right\}_{j \in\{A, B\}}$ and $\left\{I_{j}\right\}_{j \in\{A, B, C\}}$, respectively. Figure 5 shows that after three iterations of applying Eq. (1), the mean 3D ICE reduces to approximately $8 \%$ of its original value and after three iterations of applying Eq. (2) followed by Eq. (1), the mean 3D TE reduces to approximately $10 \%$ of its original value. Figure 5 also shows the percentage of voxels with ICE and TE greater than the size of one voxel $(3.73 \mathrm{~mm}$ corner to corner.) The percentage of voxels with ICE greater than one voxel was reduced from $2.6 \%$ to $0.1 \%$ and the percentage of

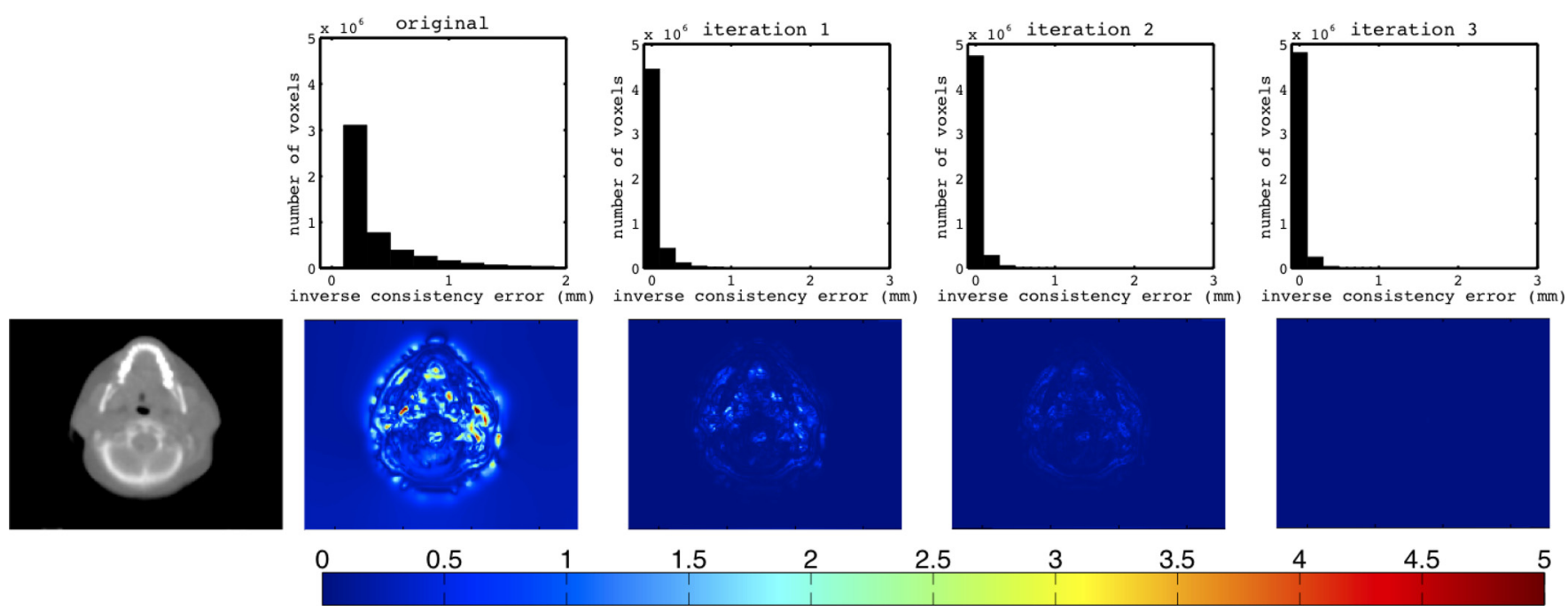

FIG. 3. ICE histograms and maps for the DVFs for the H\&N image set $\left\{I_{j}\right\}_{j \in\{A, B\}}$. Each successive histogram and map shows the $3 \mathrm{D}$ vector ICE for a successive iteration of Eq. (1). The colormap scale is in the units of mm. The image slice from which these maps are taken is shown on the left for reference. 
(a)
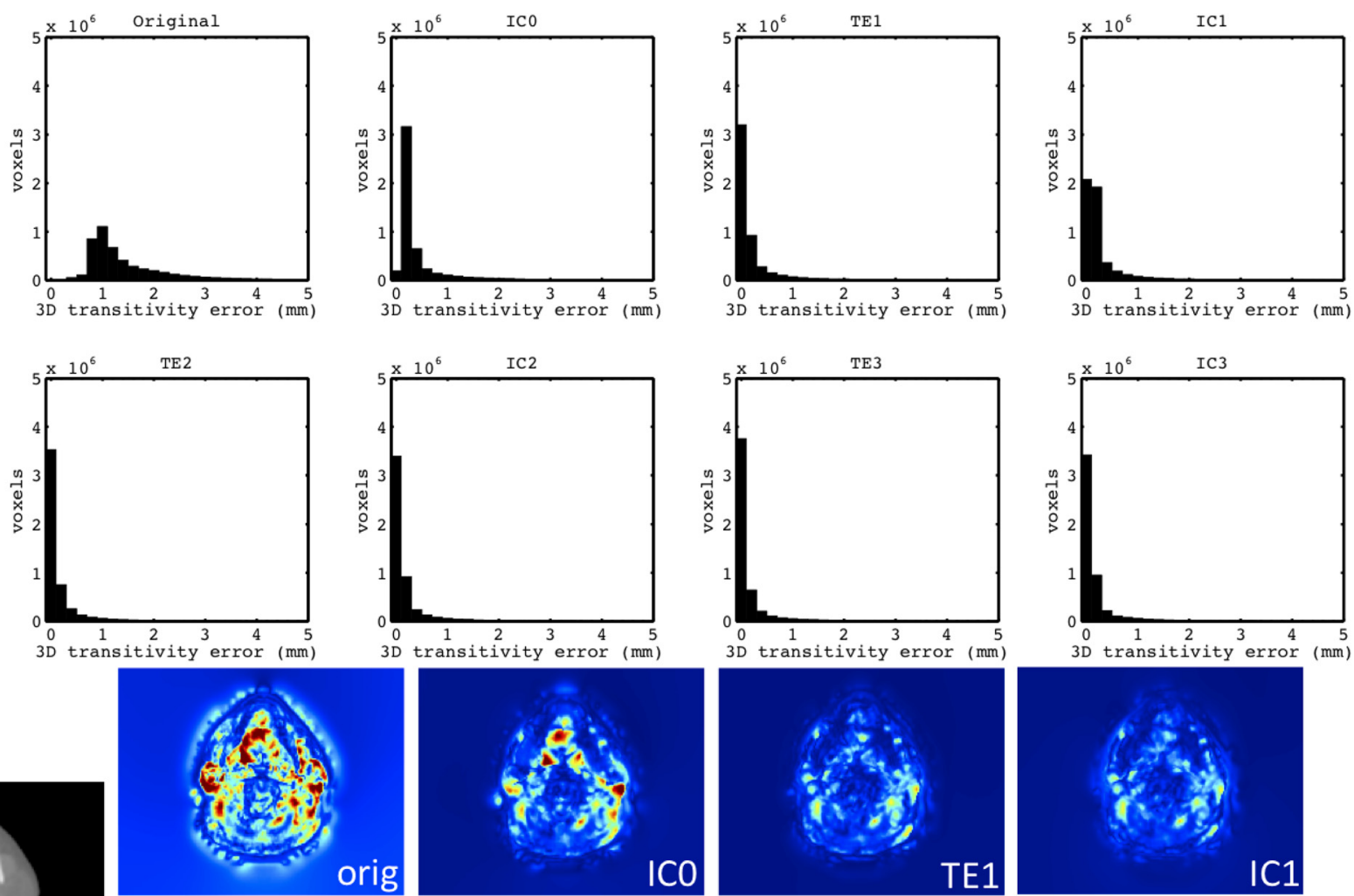

(b)
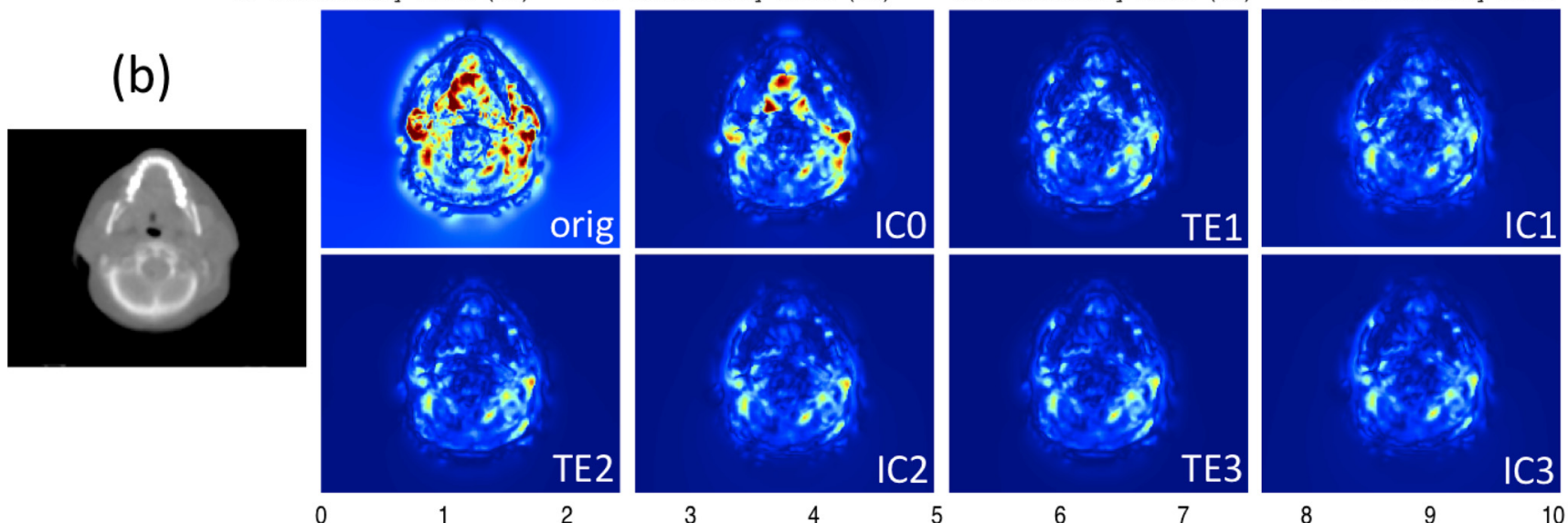

2

34 5

6

TE3

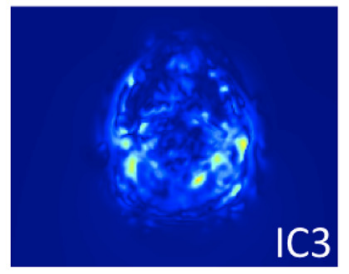

8

FIG. 4. (a) TE histograms and (b) TE maps for the H\&N images showing the reduction of TE with each successive application of Eq. (2) followed by Eq. (1). Note that our technique does not entirely remove the TE but does lead to a significant reduction in TE. The colormap scale in (b) is in the units of mm. The image slice from which these maps are taken is shown on the left for reference.
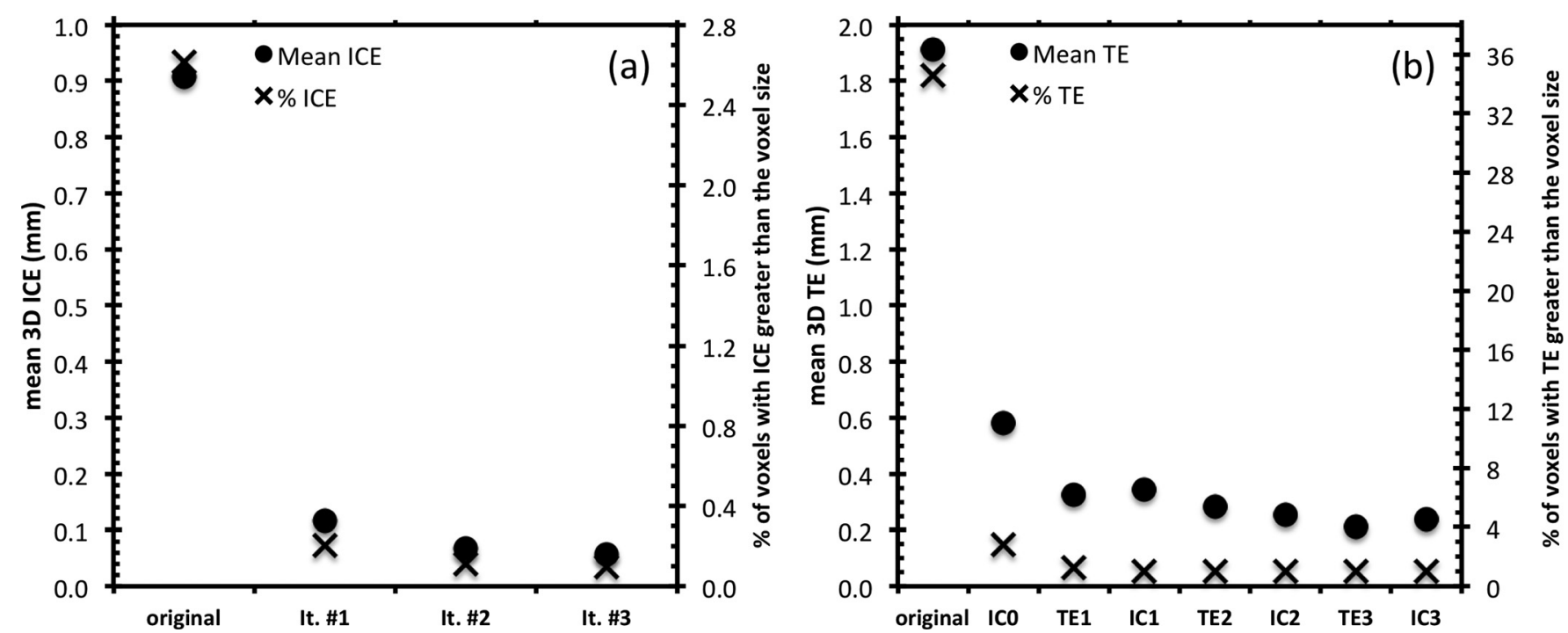

FIG. 5. (a) Mean 3D ICE and the percentage of voxels with an ICE greater than the voxel size for the image sets $\left\{I_{j}\right\}_{j \in\{A, B\}}$ for each successive application of Eq. (1) (b) Mean 3D TE and the percentage of voxels with a TE greater than the voxel size for the image set $\left\{I_{j}\right\}_{j \in\{A, B, C\}}$ for each successive iteration of Eq. (2) followed by Eq. (1). 

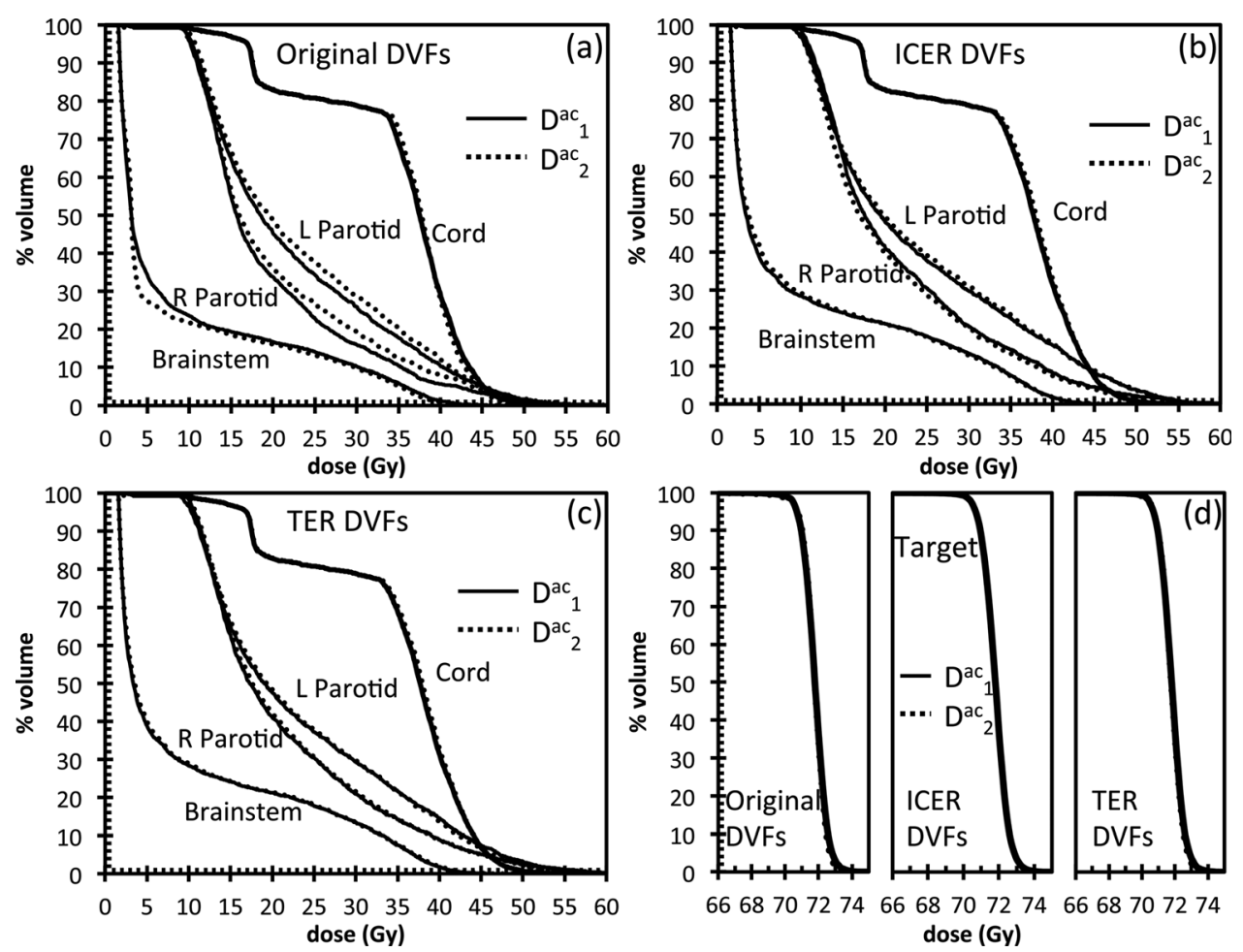

FIG. 6. OAR and target DVHs for the three sets of accumulated doses on $I_{D}$ for the head and neck MVCT data set.

voxels with TE greater than one voxel was reduced from $34.6 \%$ to $1.0 \%$. The results for the lung 4DCT data set (not shown) are similar; the mean ICE was reduced from approximately $0.81 \mathrm{~mm}$ to $0.05 \mathrm{~mm}$ and the mean TE was reduced from approximately $1.35 \mathrm{~mm}$ to $0.44 \mathrm{~mm}$, the percentage of voxels with ICE greater than one voxel was reduced from $2.7 \%$ to $4.9 \times 10^{-5} \%$ and the percentage of voxels with TE greater than one voxel was reduced from $11.1 \%$ to $1.1 \%$.

The accumulated doses $D^{a c}{ }_{1}$ and $D^{a c}{ }_{2}$, calculated using the original, ICER and TER DVFs were compared for both data sets. The target and OAR DVHs are shown in Fig. 6. For the target, no difference is observed in the DVHs. For the OARs however, there is some difference between the two accumulated doses. The DVHs show that with the ICER and then with TER DVFs, the agreement between the OAR DVHs for $D^{a c}{ }_{1}$ and $D^{a c}{ }_{2}$ improves. This may have a clinical impact when one looks at the mean parotid dose. Using the original DVFs, the difference in the left and right mean parotid doses between $D^{a c}{ }_{1}$ and $D^{a c}{ }_{2}$ is $0.8 \mathrm{~Gy}$ and $0.9 \mathrm{~Gy}$, respectively; using the ICER DVFs this difference reduces to $0.2 \mathrm{~Gy}$ and $0.3 \mathrm{~Gy}$; using the TER DVFs the difference reduces to $0 \mathrm{~Gy}$ and $0.1 \mathrm{~Gy}$. It must be noted that the differences in dose are not completely removed but are significantly reduced. Figure 7 shows the target and OAR DVHs for the lung 4DCT data set. Although the differences between $D^{a c}{ }_{1}$ and $D^{a c}{ }_{2}$ are not as large as for the head and neck MVCT data set, the agreement between the OAR and target DVHs for $D^{a c}{ }_{1}$ and $D^{a c}{ }_{2}$ improves with the ICER and TER DVFs.

The effect of the presented postprocessing technique on the registration accuracy for the lung 4DCT data is shown in Fig. 8. The histograms show that the influence of Eq. (1) and the iterative use of Eqs. (2) and (1) on the registration accu- racy is minimal. There is a slightly positive shift of the histogram, however, the magnitude of this shift is of the order of $0.1 \mathrm{~mm}$. The mean registration error in the three directions is presented in Table I, which shows minimal differences in the mean deformation error between original, ICER and TER DVFs. None of the results were statistically significant.

\section{DISCUSSION}

The above results show the effect on dose accumulation of inverse consistency and transitivity errors. When the dose is accumulated on the same image via different image pathways, the accumulated dose is different. The clinical implication of this inconsistency is an introduction of ambiguity into the decision-making process in adaptive radiotherapy. A postprocessing technique was introduced that significantly reduces the inverse consistency and transitivity errors. When the technique was applied to DVFs from a noninverse consistent and nontransitive algorithm, consistent dose accumulation results independent of the image pathway selected for dose accumulation were obtained.

It can be seen that the magnitude of the inconsistencies in dose accumulation, that is, the difference between $D^{a c}{ }_{1}$ and $D^{a c}{ }_{2}$, varied between the data sets used, with the lung 4DCT data set having the lesser difference. In both cases, however, we note that our technique does not entirely remove transitivity error but does yield a significant reduction in transitivity error. The TE contains contributions from all DVFs used to calculate the TE. It is unknown, however, how much each DVF contributes to the calculated TE thus there is no way to weight each of the DVFs in the postprocessing technique to take this into account. As a result, all of the DVFs received 

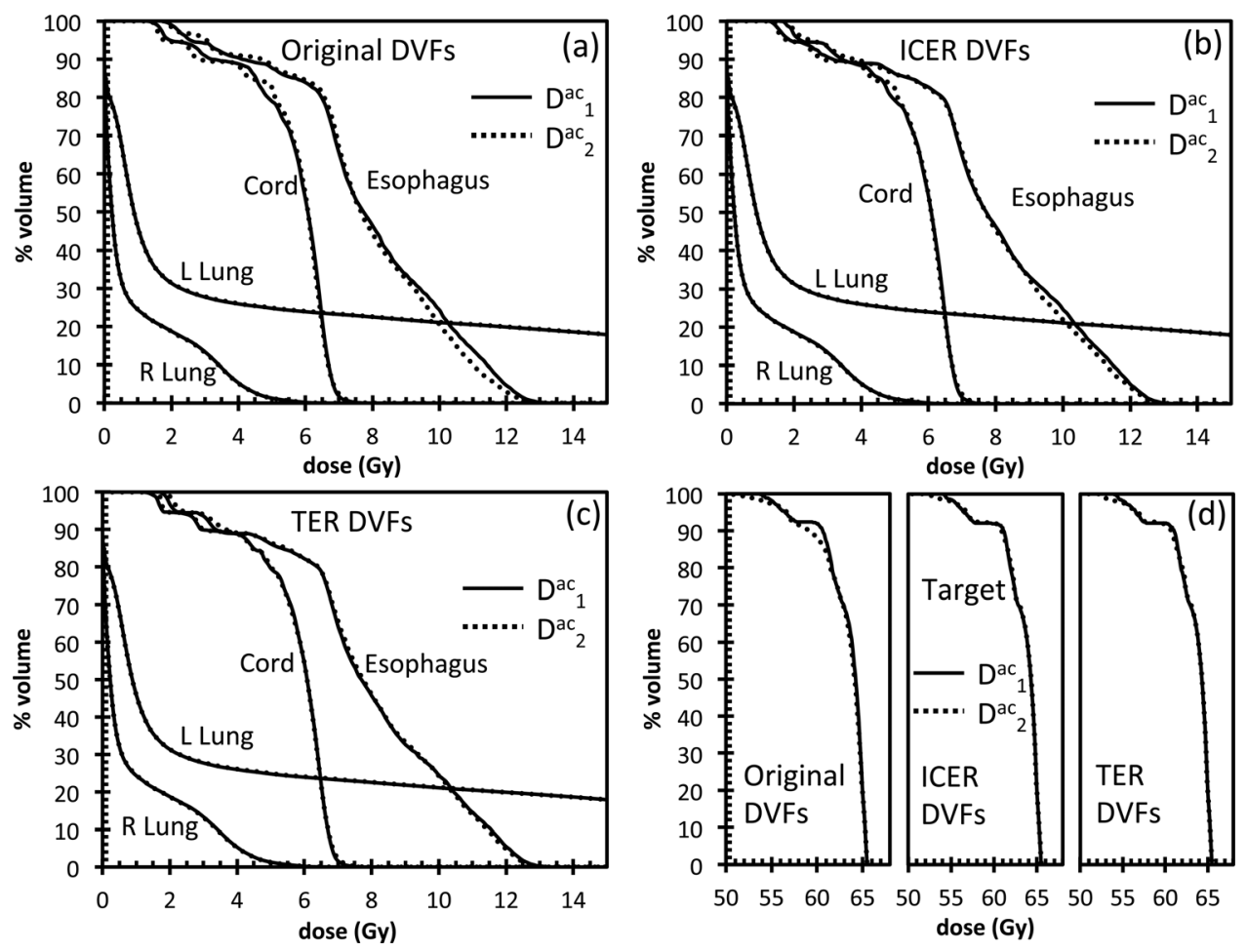

FIG. 7. OAR and target DVHs for the three sets of accumulated doses on $I_{D}$ for the Lung 4DCT data.

equal weighting to calculate the TE-reduced DVF, which may result in residual TE in the DVFs. However, in both cases, the differences in the DVHs show that the effect of the TE is effectively removed, thus allowing consistent dose accumulation. It is also true that the correction method stated relates only to differences due to inverse and transitivity inconsistencies and does not significantly affect the accuracy of the deformation. Figure 8 and Table I show minimal effect of the postprocessing technique on the registration

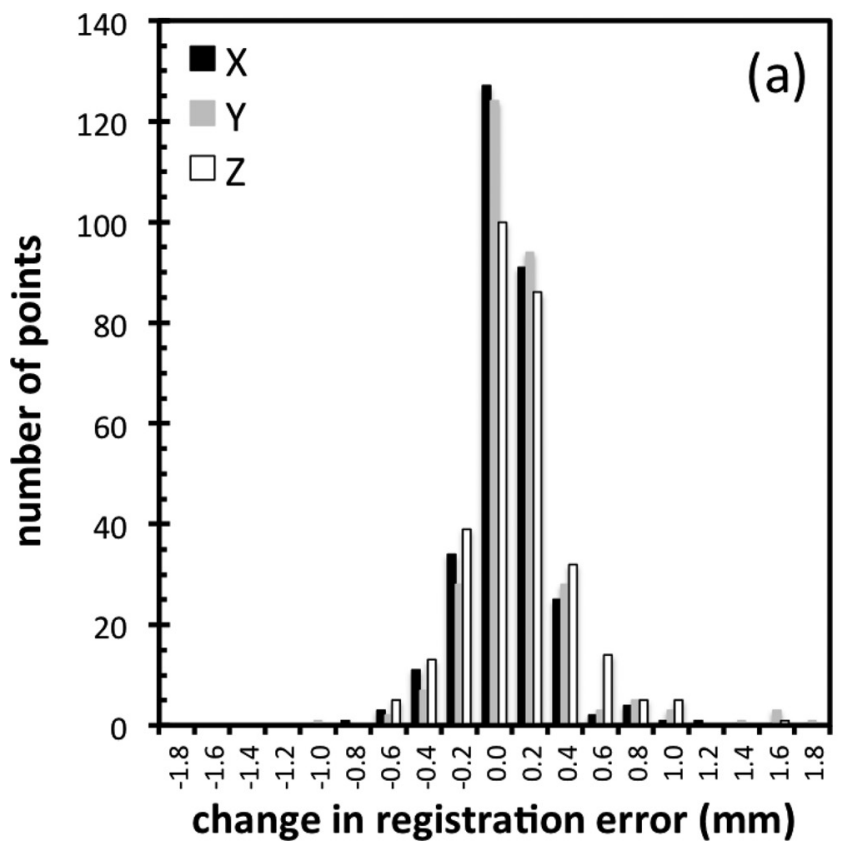

accuracy for the lung 4DCT data set. That is to say, the dose accumulation for the lung 4DCT data set was more consistent, without significant loss in registration accuracy.

In its current implementation, the method is timeconsuming as it requires computationally intensive DVF inversion. However, in practice, only one extra image at a time will be available during the radiotherapy course, therefore, reducing inverse consistency and transitivity errors is only required for one extra image at any one time, as

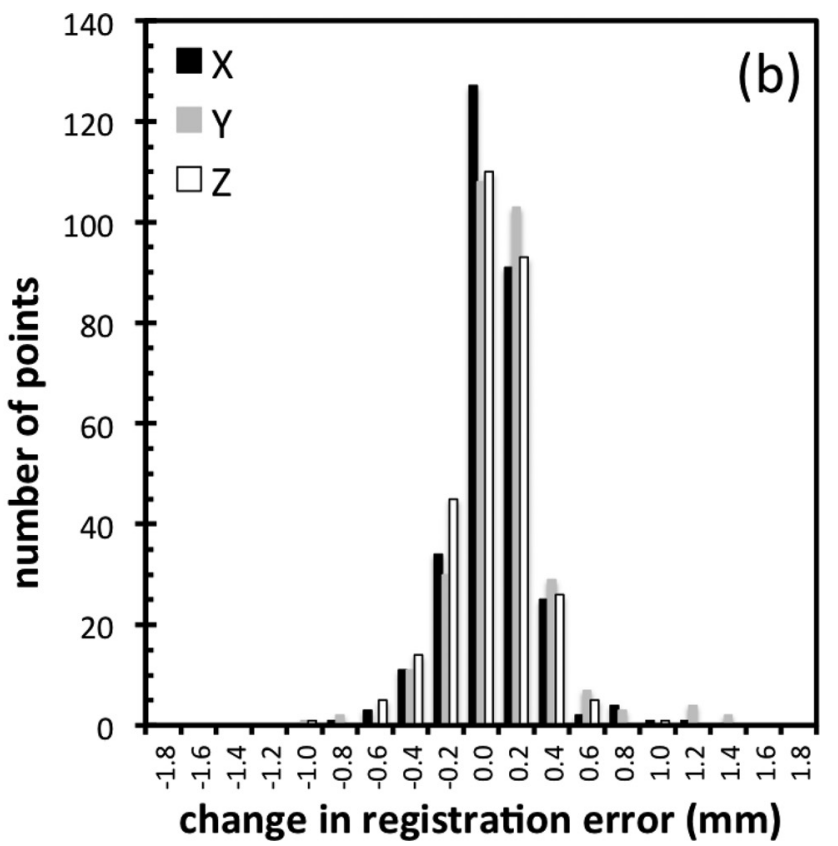

FIG. 8. Change in the registration error for the lung 4DCT data from the original DVFs after (a) ICER DVFs (b) TER DVFs. A negative change means that the registration error was reduced with postprocessing. A positive change means that the registration error was increased with postprocessing. 
TABLE I. Mean registration error of the original, ICER and TER DVFs for the lung 4DCT data. The values given are the mean \pm 1 S.D. The $p$ value from the ANOVA test is given for the processed DVF results.

\begin{tabular}{lccc}
\hline \hline Direction & Original $(\mathrm{mm})$ & $\operatorname{ICER}(\mathrm{mm})$ & $\operatorname{TER}(\mathrm{mm})$ \\
\hline $\mathrm{x}$ & $0.47 \pm 0.40$ & $0.44 \pm 0.42(p=0.41)$ & $0.42 \pm 0.42(p=0.12)$ \\
$\mathrm{y}$ & $0.48 \pm 0.42$ & $0.50 \pm 0.51(p=0.53)$ & $0.49 \pm 0.49(p=0.76)$ \\
$\mathrm{z}$ & $0.87 \pm 0.60$ & $0.89 \pm 0.68(p=0.74)$ & $0.88 \pm 0.70(p=0.90)$ \\
\hline
\end{tabular}

opposed to all of the available images at once as done for this study. This could significantly reduce the computation time required at any one time by allowing some distribution of computation over the course of the treatment. The initial DVF inversion steps could possibly be removed if an inverse consistent algorithm was used for the generation of the initial DVFs, which could reduce the computation time.

Although the presented post processing technique leads to a consistent accumulated dose for a given set of images considering all deformation pathways, an investigation into the accuracy of the resulting accumulated dose based on ICER and TER DVFs versus the original DVFs is beyond the scope of our current study. This topic will be addressed in future work.

\section{CONCLUSION}

Dose accumulation from multiple image sets to one image is dependent on the image pathway via which the dose is accumulated, introducing discrepancies between doses accumulated on the same image via different image pathways. This study presents a postprocessing method to reduce this difference by reducing inverse consistency and transitivity errors between all DVFs used in dose accumulation. The results show that DVFs processed to reduce inverse consistency error improve the agreement between doses accumulated along different image pathways. This agreement is further improved using DVFs processed to reduce transitivity error. The presented postprocessing technique has minimal effect on the registration accuracy, however, any changes in dose accumulation accuracy using this method is still to be determined in a future study.

\section{ACKNOWLEDGMENTS}

The work of this manuscript was a joint effort from all authors, with the exception of the proposition and lemma in the Appendix, which was specifically contributed by W. A. Tomé. E. T. Bender and N. Hardcastle contributed equally to this work.

\section{APPENDIX: THIRD ORDER IMAGE TRANSITIVITY IMPLIES HIGHER ORDER IMAGE TRANSITIVITY}

A mathematical proof that a set of inverse consistent mappings that is not subject to three-image transitivity errors is also not subject to four-image or higher transitivity errors is given below.

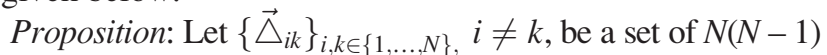
deformation mappings between $N$ image sets $\left\{I_{k}\right\}_{k \in\{1, \ldots, N\}}$ that is inverse consistent and three-image transitive. That is, $\vec{\triangle}_{k i} \circ \vec{\triangle}_{i k}=\hat{1}, i \neq k$ and $\vec{\triangle}_{k i} \circ \vec{\triangle}_{j k} \circ \vec{\triangle}_{i j}=\hat{1}, i \neq j \neq k$. Any composition of two distinct maps $\vec{\triangle}_{s k} \circ \vec{\triangle}_{i s}, i \neq k \neq s$ from the set of mappings $\left\{\vec{\triangle}_{i k}\right\}_{i, k \in\{1, \ldots, N\}}$ between any three distinct image sets $I_{i}, I_{s}$, and $I_{k}$ is then equivalent to $\vec{\triangle}_{i k}, i \neq k$.

Proof: Applying $\vec{\triangle}_{j i}, i \neq j$ from the right to $\vec{\triangle}_{k i} \circ \vec{\triangle}_{j k}$ $\circ \vec{\triangle}_{i j}=\hat{1}, i \neq j \neq k$, yields $\left(\vec{\triangle}_{k i} \circ \vec{\triangle}_{j k} \circ \vec{\triangle}_{i j}\right) \circ \vec{\triangle}_{j i}=\hat{1} \circ \vec{\triangle}_{j i}$, $i \neq j \neq k$. Using the associative property and the fact that the set of maps is inverse consistent, this simplifies to $\vec{\triangle}_{k i} \circ \vec{\triangle}_{j k}=\vec{\triangle}_{j i}, i \neq j \neq k$.

Lemma: If a set $\left\{\vec{\triangle}_{i k}\right\}_{i, k \in\{1, \ldots, N\}}, i \neq k$, of $N(N-1)$ deformation mappings between $N$ image sets $\left\{I_{k}\right\}_{k \in\{1, \ldots, N\}}$ is inverse consistent and three-image transitive, then the set of mappings $\left\{\vec{\triangle}_{i k}\right\}_{i, k \in\{1, \ldots, N\}}, i \neq k$, is also not subject to higher order image transitivity errors. That is, let $\left\{I_{j(v)}\right\}_{j(v) \in\{1, \ldots, k\}}$ be an arbitrary subset of $\mathrm{k}>3$ distinct images, then

$$
\begin{aligned}
& \vec{\triangle}_{i(v) j(v)} \circ \vec{\triangle}_{j(v) m(v)} \circ \ldots \circ \vec{\triangle}_{u(v) v(v)}=\vec{\triangle}_{i(v) v(v)}, \\
& i(v) \neq j(v) \neq m(v) \neq \ldots \neq u(v) \neq v(v)
\end{aligned}
$$

Proof: Let us choose any arbitrary subset of $k>3$ distinct images $\left\{I_{j(v)}\right\}_{j(v) \in\{1, \ldots, k\}}$ from the set of $N$ images $\left\{I_{j}\right\}_{j \in\{1, \ldots, N\}}$ along with their corresponding $k(k-1)$ deformation maps $\left\{\vec{\triangle}_{i(v) j(v)}\right\}_{i(v), j(v) \in\{1, \ldots, k\}}, i(v) \neq j(v)$, then we can form the following $k$ image deformation chain:

$$
\begin{aligned}
& I_{i(v)} \stackrel{\vec{\Delta}_{i(v) j(v)}}{\longrightarrow} I_{j(v)} \stackrel{\vec{\Delta}_{j(v) m(v)}}{\longrightarrow} I_{m(v)} \ldots I_{n(v)} \stackrel{\vec{\Delta}_{n(v) r(v)}}{\longrightarrow} I_{r(v)}, \\
& i(v) \neq j(v) \neq m(v) \neq \cdots \neq n(v) \neq r(v)
\end{aligned}
$$

Since, by assumption the set $\left\{\vec{\triangle}_{i(v) j(v)}\right\}_{i(v), j(v) \in\{1, \ldots, k\}}$, $i(v) \neq j(v)$ is three-image transitive, we can eliminate image sets in any order in the above chain between $I_{i(v)}$ and $I_{r(v)}$ by replacing the corresponding pair of deformation maps with the deformation map relating the immediate prior image set and the immediate following image set to the one being eliminated. Suppose that we choose in the first step to eliminate image set $I_{j(v)}$ then we arrive at

$$
\begin{aligned}
& I_{i(v)} \stackrel{\vec{\triangle}_{i(v) m(v)}}{\longrightarrow} I_{m}(v) \ldots I_{n(v)} \stackrel{\vec{\triangle}_{n(v) r(v)}}{\longrightarrow} I_{r(v)}, \\
& i(v) \neq m(v) \neq \cdots \neq n(v) \neq r(v) .
\end{aligned}
$$

We can continue this process in any order $k-4$ more times and finally arrive at

$$
I_{i(v)} \stackrel{\vec{\triangle}_{i(v) q(v)}}{\longrightarrow} I_{q(v)} \stackrel{\vec{\Delta}_{q(v) r(v)}}{\longrightarrow} I_{r(v)}, \quad i(v) \neq q(v) \neq r(v)
$$

which shows that the arbitrary subset of mappings $\left\{\vec{\triangle}_{i(v), j(v)}\right\}_{i(v), j(v) \in\{1, \ldots, k\}}, i(v) \neq j(v)$ is not subject to $k$-image transitivity errors. Since $k$ above was arbitrary this is true for all $3<k \leq N$ proving the assertion that a set of deformation mappings that is inverse consistent and three-image transitive is also not subject to higher order image transitivity errors.

${ }^{\text {a)} E l e c t r o n i c ~ m a i l: ~ t o m e @ h u m o n c . w i s c . e d u ~}$

${ }^{1}$ E. T. Bender and W. A. Tomé, "The utilization of consistency metrics for error analysis in deformable image registration," Phys. Med. Biol. 54, (18), 5561 (2009). 
${ }^{2}$ G. E. Christensen and H. J. Johnson, "Consistent image registration," IEEE Trans. Med. Imaging 20, 568-582 (2001).

${ }^{3}$ G. E. Christensen and H. J. Johnson, "Invertibility and transitivity analysis for nonrigid image registration," J. Electron. Imaging 12, 106-117 (2001).

${ }^{4} \mathrm{X}$. Geng, D. Kumar, and G. E. Christensen, "Transitive inverseconsistent manifold registration," Inf. Process Med. Imaging 19, $468-479$ (2005).

${ }^{5}$ A. Leow, S. C. Huang, A. Geng, J. Becker, S. Davis, A. Toga, and P. Thompson, "Inverse consistent mapping in 3D deformable image registration: Its construction and statistical properties," Inf. Process Med. Imaging 19, 493-503 (2007).

${ }^{6}$ D. Yang, H. Li, D. A. Low, J. O. Deasy, and I. El Naqa, "A fast inverse consistent deformable image registration method based on symmetric optical flow computation," Phys. Med. Biol. 53, 6143-6165 (2008).
${ }^{7}$ O. Skrinjar, A. Bistoquet, and H. Tagare, "Symmetric and transitive registration of image sequences,” Int. J. Biomed. Imaging 2008, 686875 (2008).

${ }^{8}$ T. Vercauteren, X. Pennec, A. Perchant, and N. Ayache, "Diffeomorphic demons using ITK's finite difference solver hierarchy," The Insight Journal 2007 MICCAI Open Science Workshop, 2007, http://hdl.handle.net/ $1926 / 510$

${ }^{9}$ R. Castillo, E. Castillo, R. Guerra, V. E. Johnson, T. McPhail, A. K. Garg, and T. Guerrero, "A framework for evaluation of deformable image registration spatial accuracy using large landmark point sets," Phys. Med. Biol. 54, 1849-1870 (2009).

${ }^{10} \mathrm{P}$. Dupuis, U. Grenander, and M. I. Miller, "Variational problems on flows of diffeomorphisms for image matching," Quart. Appl. Math. 56(3) 587600 (1998).

${ }^{11}$ T. Vercauteren, X. Pennec, A. Perchant, and N. Ayache, "Diffeomorphic demons: Efficient non-parametric image registration," Neuroimage 45(1 supp.) S61-72 (2009). 